\title{
The First Experimental Chain at NAL: Collaborating Despite Global Differences
}

Vitaly Pronskikh

Fermilab

APS April Meeting 2021

Reaching Out to Broader Audiences

April 18, 2021

The views and work contained herein are my own and do not reflect those of Fermilab, FRA, or U.S. DOE 


\section{First experimental chain at the NAL Main Ring Accelerator}

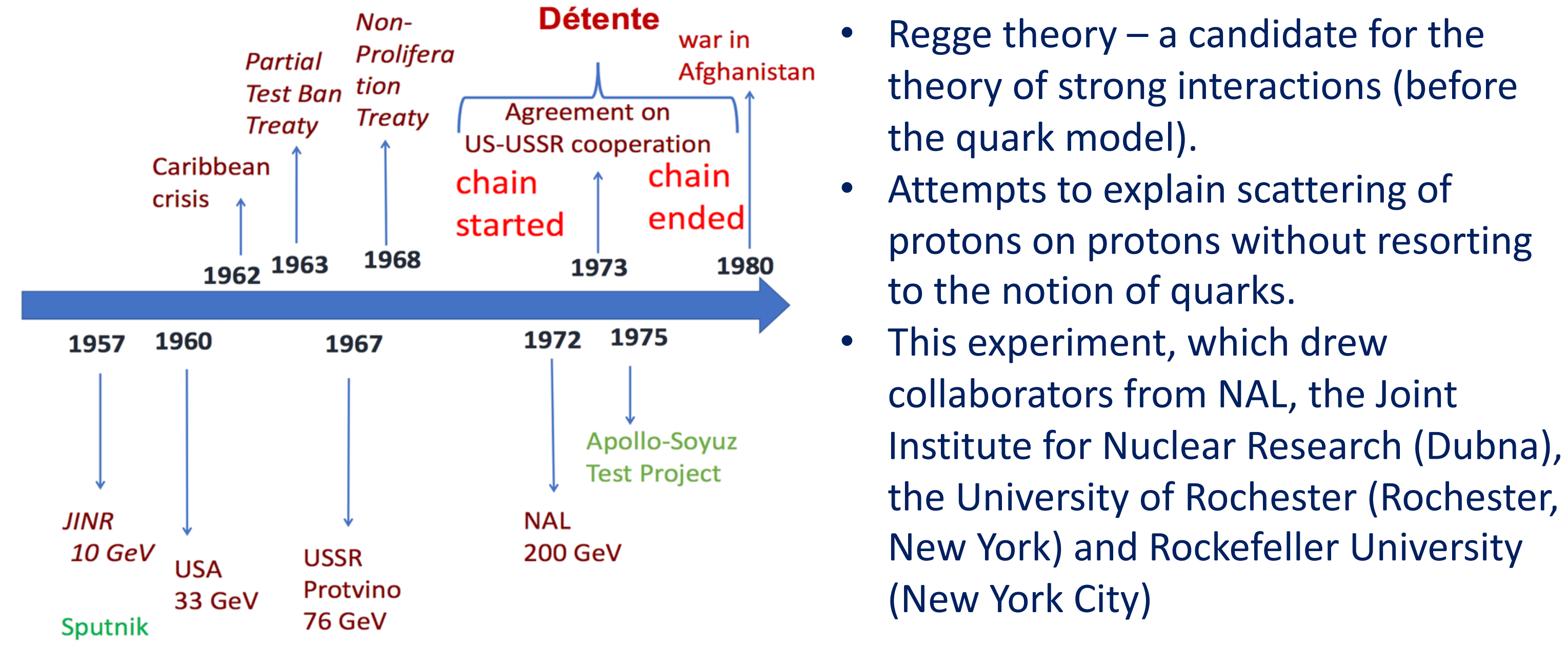




\section{The beginning of the chain}

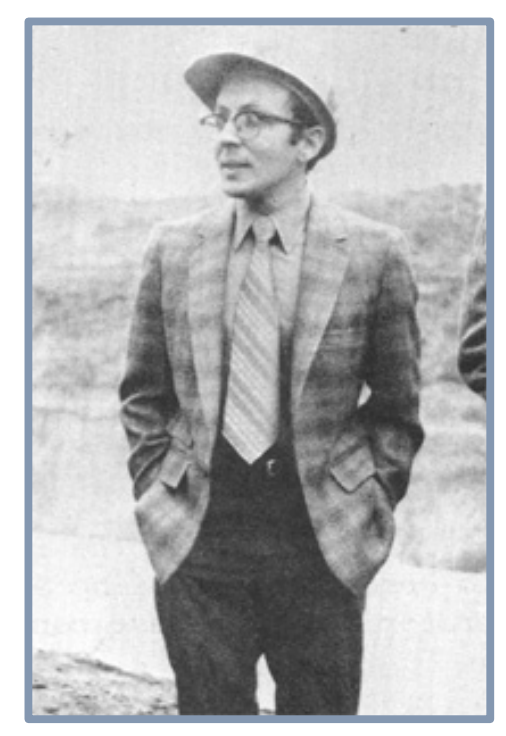

E. Malamud

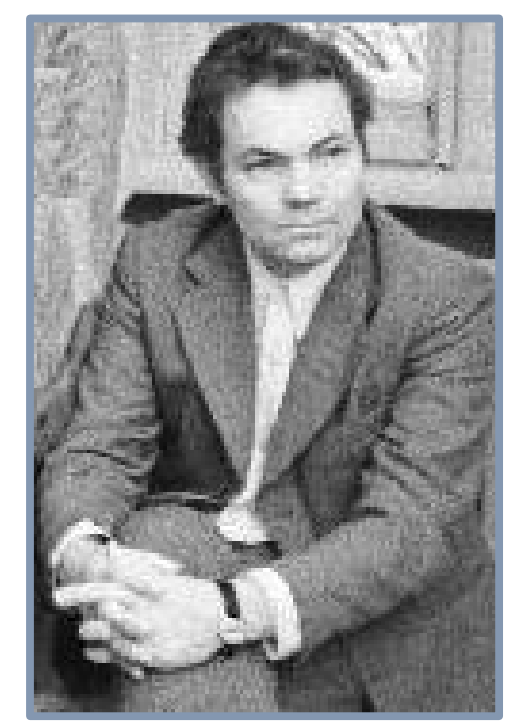

V.A.Nikitin

- R.R.Wilson encourages the idea

- G.Seaborg invites the group to Batavia

- The JINR team builds the target for NAL - Malamud and Nikitin Joint experiments started at 1972

The first experiment at NAL! Formative years of the network

- Nikitin's group from JINR conducted experiments at Protvino at the $70-\mathrm{GeV}$ accelerator in 1960s with a Tolstov-type target

- Used a unique supersonic gas jet target with cryopumping

- Results presented at ICHEP in Kiev in 1970. Received a big attention. agreed to collaborate and propose an experiment at NAL

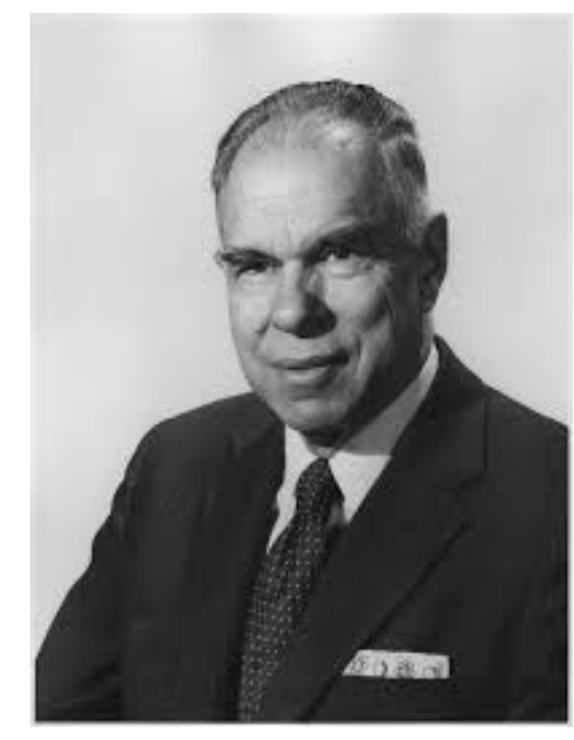

G. Seaborg

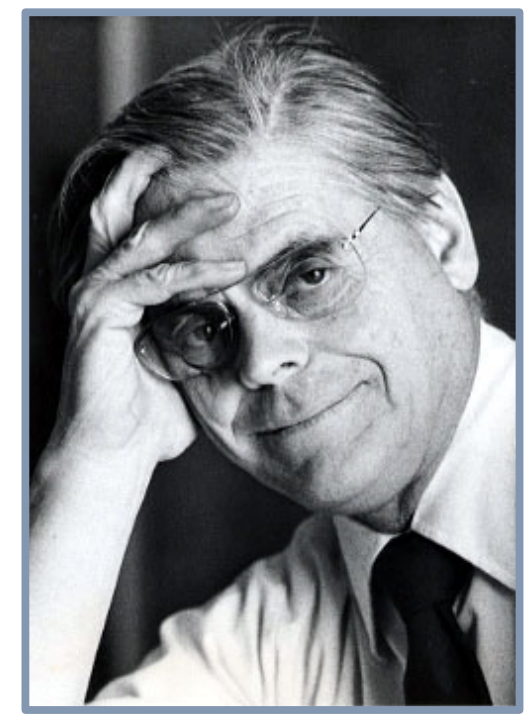

R.R.Wilson 


\section{Trading zones and actor-network approach}

- A trading zone:

- Explicit: a trading zone is a place where an exchange between cultures occurs (Gorman 2010).

- Implicit definition: a trading zone can be understood as the unity of the social, technical, and theoretical components in which the exchange interaction between epistemic cultures is situated.

- Actor-Network Theory (Latour 2013): scientific knowledge is represented as a product of assembly, constructed in the course of the interaction of a number of actors, which can be both living (scientists, engineers) and inanimate (devices, gas targets). It becomes impossible to separate content from context in them and to discern scientific facts from their external background.

- Collective experimentalist: a network of actors; form of the network: trading zones. 


\section{Chain develops as a self-sustained trading zone}

- The jet quickly became a users facility: B.Maglic's experiment used it simultaneously for different physics program.

- E-67. Spokesman B.Maglic. Searches for new particles (baryon resonances) and discovered two resonances.

- The US-USSR collaborators set up and ran the target for the Maglic group. The Maglic group received the target parameters and analyzed the data. Thus, the Dubna/NAL group provided the target as a service, without training the E-67 team. Boundary object.

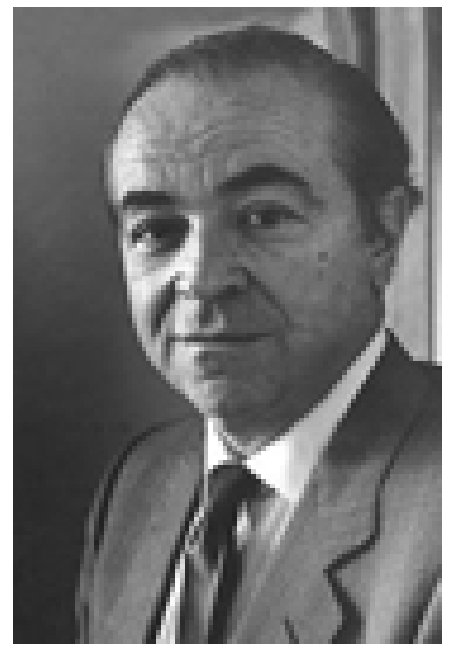

B.Maglic

- From 1971 to 1976, Paolo Franzini and Juliet Lee-Franzini were actively involved in diffraction physics research at NAL. Their E-14 project, running from May 1971 to June 1973 (Franzini 1970), like the experiment of the Malamud-Nikitin group, was within the framework of the Regge and Pomeranchuk theories.

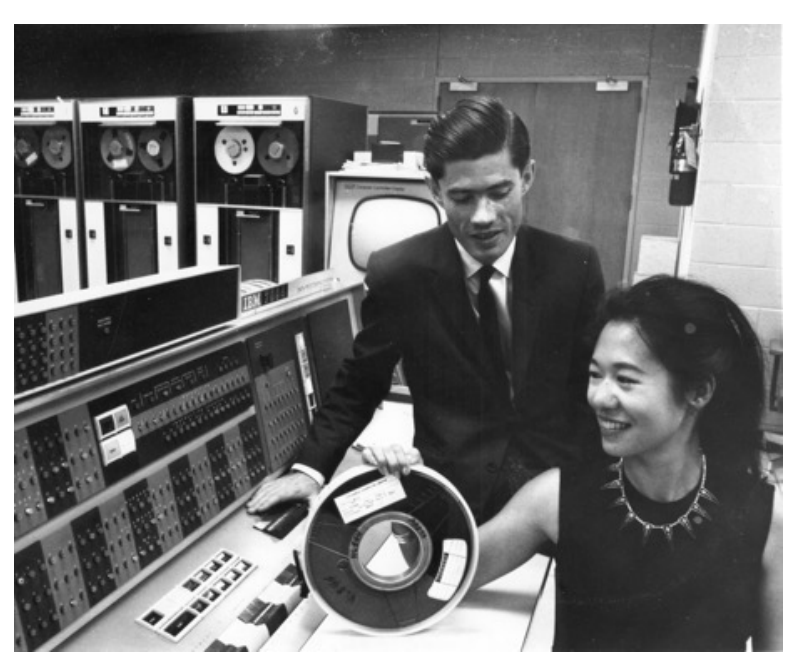


Encouraged and self-sustained states of the chain

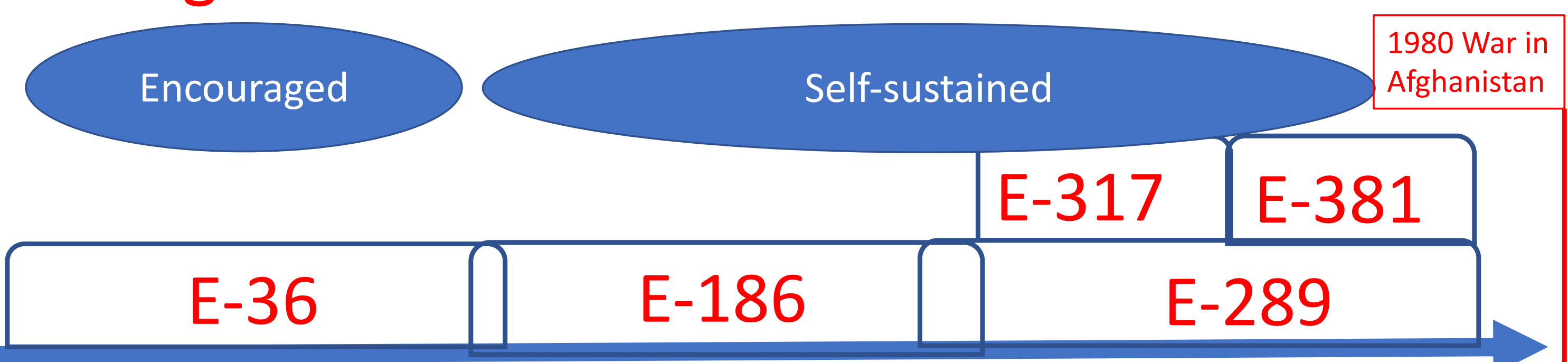

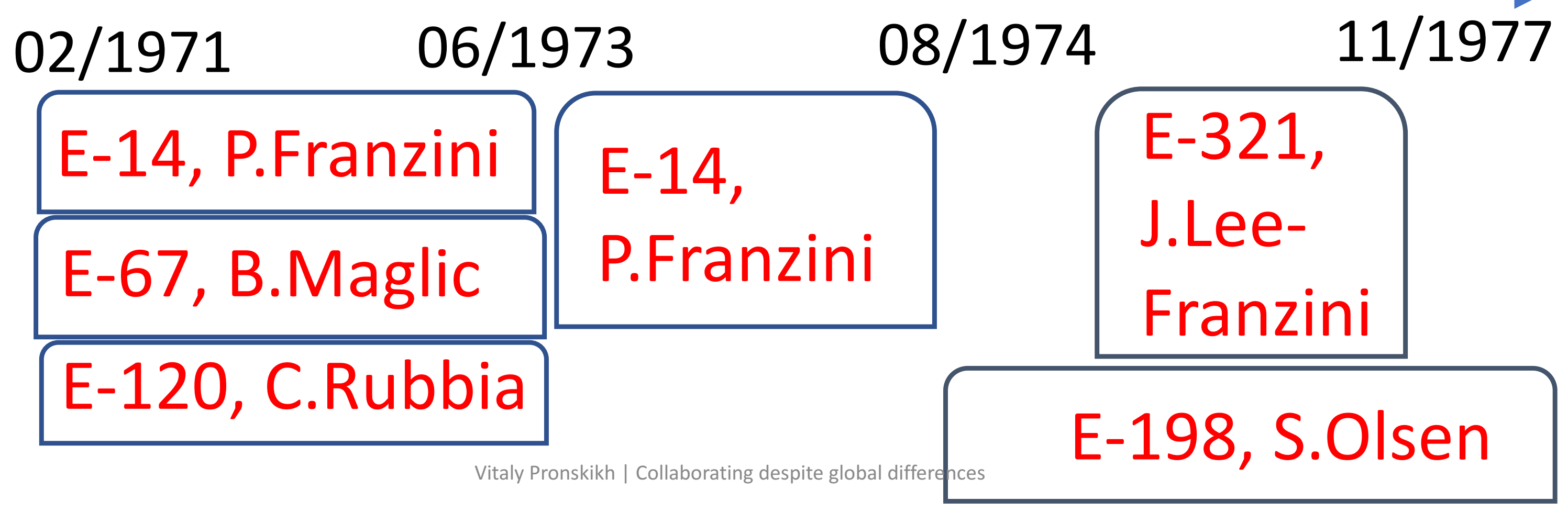




\section{Conclusions}

- The actor-network theory and the trading zone concept enable us to consider the US-USSR experimental chain as a subsequence of forms of collective experimentalists that included scientists, scientific-political actors (such as Wilson and Seaborg), and inanimate actors (such as gas jet targets).

- As forms of social life arranged around large-scale apparatuses, such experiments are even able to survive paradigmatic shifts in theory, such as the 1974 November Revolution.

- The model of trading zones suggests an effective strategy for creating small but stable international collaborations, and the model allows us to predict possible trajectories of future collaborative development.

- International collaborations require arrangements and strong institutional attention only at their early stages; to self-sustain, grow, and develop, they must be free of interference and artificial hurdles. 